\title{
A Mathematical Model of a Tuberculosis Transmission Dynamics Incorporating First and Second Line Treatment
}

\section{${ }^{* 1,7}$ ANDRAWUS, J; ${ }^{1}$ EGUDA, FY ; ${ }^{2}$ USMAN, IG; ${ }^{3}$ MAIWA, SI; ${ }^{4}$ DIBAL, IM; ${ }^{5}$ URUM, TG; ${ }^{6,7}$ ANKA, GH}

\author{
${ }^{* 1}$ Department of Mathematics, Federal University Dutse, Jigawa State, Nigeria \\ ${ }^{2}$ Department of Mathematics, Zamfara State College of Education, Maru. Zamfara State, Nigeria \\ ${ }^{3}$ Department of Mathematics, Federal University Birnin Kebbi, Kebbi State, Nigeria \\ ${ }^{4}$ Department of Statistics, Federal Polytechnic Damaturu, Yobe State, Nigeria \\ ${ }^{5}$ Department of Mathematics, Moddibo Adama University of Technology Yola, Adamawa State \\ ${ }^{6}$ Department of Mathematics, Federal University Gusau, Zamfara State, Nigeria \\ ${ }^{7}$ Department of Mathematics, Kebbi State University of Science and Technology Aliero, Kebbi State, Nigeria \\ *Corresponding Author Email: jamesany7772@gmail.com
}

\begin{abstract}
This paper presents a new mathematical model of a tuberculosis transmission dynamics incorporating first and second line treatment. We calculated a control reproduction number which plays a vital role in biomathematics. The model consists of two equilibrium points namely disease free equilibrium and endemic equilibrium point, it has been shown that the disease free equilibrium point was locally asymptotically stable if the control reproduction number is less than one and also the endemic equilibrium point was locally asymptotically stable if the control reproduction number is greater than one. Numerical simulation was carried out which supported the analytical results.
\end{abstract}

DOI: https://dx.doi.org/10.4314/jasem.v24i5.29

Copyright: Copyright $(0) 2020$ Andrawus et al. This is an open access article distributed under the Creative Commons Attribution License (CCL), which permits unrestricted use, distribution, and reproduction in any medium, provided the original work is properly cited.

Dates: Received: 12 March 2020; Revised: 30 April 2020; Accepted: 26 May 2020

Keywords: Mathematical Model, Biomathematics, Reproduction Number, Disease Free Equilibrium, Endemic Equilibrium Point

Tuberculosis (TB) is a protracted bacterial infectious disease caused by Mycobacterium tuberculosis which position a major health, social and economic burden globally, especially in low and middle income countries (WHO, 2013). The surge in HIV-TB coinfection and growing emergence of multidrugresistant TB (MDR-TB) and extensively drug resistant TB (XDR-TB) strains has further fuelled TB epidemic (WHO,2013; WHO, 2016). It is the second fatal disease due to a single infectious agent only after HIV/AIDS (WHO, 2013; WHO, 2016). TB usually affects the lungs but it can also affect other sites as well (extra-pulmonary TB). Tuberculosis is conveyed by tiny airborne droplets which are ejected into the air when a person with active pulmonary TB coughs or talks (Issarowa et al., 2015). According to the World Health Organization (WHO), in 2013, about 9million people were infected, worldwide, with $\mathrm{TB}$ and 1.5 million deaths from the disease were reported, 360,000 of whom were HIV-positive (Yang et al., 2014). Tuberculosis is seen to be declining slowly each year and an estimated 37 million lives were saved between 2000 and 2013 through effective diagnosis and treatment (Yang et al., 2014; Okuonghae and
Ikhimwin, 2016). Numerous mathematical models have been developed and used to study the transmission dynamics of TB in a population (Aparicio and Castillo-Chavez, 2009; Castillo-Chavez and Song, 2004; Feng, 2000). For instance, Okuonghae (2013) worked on a deterministic TB model with genetic heterogeneity in susceptibility and disease progression. Zhang and Feng (2000) constructed and analyzed a dynamical model to investigate the spread of TB in a community with isolation and incomplete treatment. The purpose of this article is to formulate a mathematical model of a tuberculosis transmission dynamics incorporating first and second line treatment which provides insights into the drug resistant cases in first and second line treatment. The model considers human population $N$ . The population at time $t$ is divided into six (6) subpopulations. Susceptible $(S)$ : this class include those individuals who are at risk for developing an infection from Tuberculosis (TB). Latent Class $(E)$ : this class refers to susceptible individuals who become infected. Infected $(I)$ : this class includes all 
individuals who are showing the symptom of the disease. First Line Treatment $\left(\mathrm{R}_{1}\right)$ : this class include all individual that failed to take drugs (treatment). Second Line Treatment $\left(\mathrm{R}_{2}\right)$ : this class include all individual that failed to take drugs (treatment) for the second treatment term. Recovered $(\mathrm{R})$ : this class include all individual that have recovered from the disease and got temporary immunity. The susceptible class is increased by birth or emigration at the rate of $\Lambda$. The susceptible class will get TB when they mingled with infectious individuals at the $\lambda$, where $\lambda=\beta I S / N, \beta$ is the effective contact rate. The latent class is generated at a rate $\lambda=\beta I S / N$, it decrease at rate $\sigma$ which is the progression rate. The Infectious class is generated at a rate $\sigma$, it also reduces at rate of treatment $\tau_{1}$ and it further reduce at a rate $\gamma_{1}$ which is default of treatment. The first line treatment is generated at rate $\gamma_{1}$, it reduces at a rate of treatment $\tau_{2}$ and it further reduce at a rate $\gamma_{2}$ which is second default of treatment. The second line treatment class is generated at rate $\gamma_{2}$, it reduces at a rate of treatment $\tau_{3}$ and recovered class is generated at rate $\tau_{1}, \tau_{2}$ and $\tau_{3}$. The Infectious, First line treatment and Second line treatment class are reduced at the rates $\delta_{1}, \delta_{2}$ and $\delta_{3}$ while the whole classes reduces at the rate $\mu$ which is the natural mortality rate. Hence we have that

$N(t)=S(t)+E(t)+I(t)+R_{1}(t)+R_{2}(t)+R(t)$

The above assumptions result in the following system of nonlinear ordinary differential equations:

$$
\begin{aligned}
& \frac{d S}{d t}=\Lambda-\frac{\beta S I}{N}-\mu S \\
& \frac{d E}{d t}=\frac{\beta S I}{N}-(\mu+\sigma) E \\
& \frac{d I}{d t}=\sigma E-\left(\gamma_{1}+\tau_{1}+\mu+\delta_{1}\right) \mathrm{I} \\
& \frac{d R_{1}}{d t}==\gamma_{1} I-\left(\gamma_{2}+\tau_{2}+\mu+\delta_{2}\right) R_{1} \\
& \frac{d R_{2}}{d t}=\gamma_{2} R_{1}-\left(\tau_{3}+\mu+\delta_{3}\right) R_{2} \\
& \frac{d R}{d t}=\tau_{1} I+\tau_{2} R_{1}+\tau_{3} R_{2}-\mu \mathrm{R}
\end{aligned}
$$

Simplifying (2), we have

$$
\left.\begin{array}{l}
\frac{d S}{d t}=\Lambda-\lambda S-\mu S \\
\frac{d E}{d t}=\lambda S-K_{1} E \\
\frac{d I}{d t}=\sigma E-K_{2} \mathrm{I} \\
\frac{d R_{1}}{d t}=\gamma_{1} I-K_{3} R_{1} \\
\frac{d R_{2}}{d t}=\gamma_{2} R_{1}-K_{4} R_{2} \\
\frac{d R}{d t}=\tau_{1} I+\tau_{2} R_{1}+\tau_{3} R_{2}-\mu \mathrm{R}
\end{array}\right\}
$$

where

$$
\begin{aligned}
& K_{1}=\mu+\sigma \\
& K_{2}=\gamma_{1}+\tau_{1}+\mu+\delta_{1} \\
& K_{3}=\gamma_{2}+\tau_{2}+\mu+\delta_{2} \\
& K_{4}=\tau_{3}+\mu+\delta_{3} \\
& \lambda=\frac{\beta I}{N}
\end{aligned}
$$

Boundedness of Solutions: Consider the region

$$
\left.D_{0}=\left\{\begin{array}{l|l}
S \\
E \\
I \\
R_{1} \\
R_{2} \\
R
\end{array}\right) \in \mathrm{R}_{+}^{6} \mid \begin{array}{l}
S \geq 0 \\
E \geq 0 \\
R_{1} \geq 0 \\
R_{2} \geq 0 \\
R \geq 0 \\
N \leq \frac{\Lambda}{\mu}
\end{array}\right\}
$$

It can be shown that the set $D_{0}$ is positively invariant and a global attractor of all positive solution of the system (1)

Lemma 1 The region $D_{0}$ is positively invariant for the system (1).

Proof: The rate of change of the total human population is give as

$$
\frac{d N}{d t}=\Lambda-\mu N-\delta_{1} I-\delta_{2} R_{1}-\delta_{3} R_{2}
$$

by standard comparison theorem,

$$
\frac{d N}{d t} \leq \Lambda-\mu N
$$

using the integrating factor method

$$
T_{h} e^{\mu_{h} t} \leq \frac{\Lambda_{h}}{\mu_{h}} e^{\mu_{h} t}+T_{h}(0)-\frac{\Lambda_{h}}{\mu_{h}}
$$


$T_{h}=T_{h}(0) e^{-\mu_{h} t}+\frac{\Lambda_{h}}{\mu_{h}}\left[1-e^{-\mu_{h} t}\right]$

If $N(0) \leq \frac{\Lambda}{\mu}$ then $N \leq \frac{\Lambda}{\mu}$ so, $D_{0}$ is a positively

invariant set under the flow described in (1). Hence, no solution path leaves through and boundary of $D_{0}$. Also, since solution paths cannot leave $D_{0}$, solutions remain non-negative for non-negative initial conditions. Solutions exist for all time t. In this region, the model (1) is said to be well posed mathematically and epidemiologically.

\section{Positivity of Solution}

Lemma 2 Let the initial data for the model (1) $S(0)>0, E(0)>0, I(0)>0, R_{1}(0)>0, R_{2}(0)>0$, and $R(0)>0$ be then the solution of $S(t), E(\mathrm{t}), I(\mathrm{t}), R_{1}(\mathrm{t}), R_{2}(\mathrm{t})$, a nd $R(\mathrm{t})$ with positive initial data will remain positive for all time $t$ $>0$

Proof: Let $t_{1}=\left\{t>0: S(0)>0, E(0)>0, I(0)>0, R_{1}(0)>0, R_{2}(0)>0, R(0)>0\right\}>0$

$\frac{d S}{d t}=\Lambda-(\lambda+\mu) S$

to solve the ODE using the integrating factor method $\frac{d}{d t}\left[S(t) \exp \left\{\mu t+\int_{0}^{t}(\lambda(\tau)+\mu(\tau)) d(\tau)\right\}\right]=\Lambda\left[\exp \left\{\mu t+\int_{0}^{t}(\lambda(\tau)+\mu(\tau)) d(\tau)\right\}\right]$ $S\left(t_{1}\right)=S(0) \exp \left\{-\mu t_{1}-\int_{0}^{t_{1}}(\lambda(\tau)+\mu(\tau)) d(\tau)\right\}$ $+\left[\exp \left\{-\mu t_{1}-\int_{0}^{t}(\lambda(\tau)+\mu(\tau)) d(\tau)\right\}\right] \int_{0}^{h} \Lambda\left[\exp \left\{\mu y+\int_{0}^{n}(\lambda(\tau)+\mu(\tau)) d(\tau)\right\}\right] d y>0$

Similarly, we can also show that, $E(\mathrm{t})>0, I(\mathrm{t})>0, R_{1}(\mathrm{t}),>0 R_{2}(\mathrm{t})>0$, a nd $R(\mathrm{t})>0$ for all the time $t>0$

Local stability of the disease-free equilibrium and Reproduction Number: The system (1) has a disease free equilibrium attained by setting the right hand side to zero and the disease classes to zero to obtain

$\Omega=\left(S^{0}, E^{0}, I^{0}, R_{1}^{0}, R_{2}^{0}, R^{0}\right)=\left(\frac{\Lambda}{\mu}, 0,0,0,0,0\right)$

Using the notation in van den Driessche and Watmough (2002) the matrices $F$ and $V$ for the new infection terms and the remaining transfer terms respectively, are respectively given as

$$
F=\left(\begin{array}{llll}
0 & \beta & 0 & 0 \\
0 & 0 & 0 & 0 \\
0 & 0 & 0 & 0 \\
0 & 0 & 0 & 0
\end{array}\right)
$$

and

$$
V=\left(\begin{array}{cccc}
K_{1} & 0 & 0 & 0 \\
-\sigma & K_{2} & 0 & 0 \\
0 & -\gamma_{1} & K_{3} & 0 \\
0 & 0 & -\gamma_{2} & K_{4}
\end{array}\right)
$$

Now, it follows that the control reproduction number is given as

$$
R_{C}=\frac{\sigma \beta}{K_{1} K_{2}}=\frac{\sigma \beta}{(\mu+\sigma)\left(\gamma_{1}+\tau_{1}+\mu+\delta_{1}\right)}
$$

'The following result is established using Theorem 2 in (van den Driessche \& Watmough, 2002).

Lemma 3: The DFE of the system (1) is locally asymptotically stable if $R_{C}<1$ and unstable if $R_{C}>1$. The value $R_{C}$ is the humans effective reproduction number since there is the presence of control strategies. The threshold quantity $R_{C}$ is the control reproduction number for the model (1). By Theorem 1 , biologically speaking, Tuberculosis is eliminated from the population when $R_{C}<1$ if the initial sizes of the populations of the model are in the region of attraction of $D_{0}$. However, the disease free equilibrium may not be globally asymptotically stable even if $R_{C}<1$ in the case when a backward bifurcation occurs. That is, there is the presence of a stable EEP co-existing with the DFE.

Existence of Endemic Equilibrium Point (EEP) of the Model: Let the endemic equilibrium point be $\xi^{* *}=\left(\mathrm{S}^{* *}, \mathrm{E}^{* *}, \mathrm{I}^{* *}, \mathrm{R}_{1}^{* *}, \mathrm{R}_{2}^{* *}, \mathrm{R}^{* *}\right)$. At steady state, solving (1) in terms of force of infection we have

$$
\begin{aligned}
& \mathrm{S}^{* *}=\frac{\Lambda}{\lambda^{* *}+\mu}, \mathrm{E}^{* *}=\frac{\lambda^{* *} \Lambda}{K_{1}\left(\lambda^{* *}+\mu\right)}, \\
& \mathrm{I}^{* *}=\frac{\lambda^{* *} \sigma \Lambda}{K_{1} \mathrm{~K}_{2}\left(\lambda^{* *}+\mu\right)}, \\
& \mathrm{R}_{1}^{* *}=\frac{\lambda^{* *} \gamma_{1} \sigma \Lambda}{K_{1} \mathrm{~K}_{2} \mathrm{~K}_{3}\left(\lambda^{* *}+\mu\right)}, \\
& \mathrm{R}_{2}^{* *}=\frac{\lambda^{* *} \gamma_{1} \gamma_{2} \sigma \Lambda}{K_{1} \mathrm{~K}_{2} \mathrm{~K}_{3} \mathrm{~K}_{4}\left(\lambda^{* *}+\mu\right)}, \\
& \mathrm{R}^{* *}=\frac{\lambda^{* *} K_{3} K_{4} \tau_{1} \sigma \Lambda+\lambda^{* *} K_{4} \tau_{2} \gamma_{1} \sigma \Lambda+\lambda^{* *} \tau_{3} \gamma_{1} \gamma_{2} \sigma \Lambda}{\mu K_{1} \mathrm{~K}_{2} \mathrm{~K}_{3} \mathrm{~K}_{4}\left(\lambda^{* *}+\mu\right)}
\end{aligned}
$$

Now,

$$
\lambda^{* *}=\frac{\beta I^{* *}}{N^{* *}}
$$


Substituting the values of $I^{* *}$ and $N^{* *}$ into (20) gives

$$
J\left(\xi^{* *}\right)=\left(\begin{array}{cccccc}
-\lambda^{* *}-\mu & 0 & 0 & 0 & 0 & 0 \\
0 & -K_{1} & 0 & 0 & 0 & 0 \\
0 & 0 & -K_{2} & 0 & 0 & 0 \\
0 & 0 & 0 & -K_{3} & 0 & 0 \\
0 & 0 & 0 & 0 & -K_{4} & 0 \\
0 & 0 & 0 & 0 & 0 & -\mu
\end{array}\right)
$$

$\lambda^{* *}\left(A_{1} \lambda^{* *}+A_{2}\right)=0$

Where

$$
A_{1}=K_{1}^{2} K_{2}^{2} K_{3} K_{4}+K_{1} K_{2} K_{3} K_{4} \delta_{1} \sigma+K_{1} K_{2} K_{3} K_{4} \delta_{2} \gamma_{1} \sigma-K_{1} K_{2} \delta_{3} \gamma_{1} \gamma_{2} \sigma \text { (22) }
$$$$
A_{2}=\mu K_{1} K_{2} K_{3} K_{4}\left(1-R_{C}\right)
$$

From (21), either

$$
\lambda^{* *}=0
$$

or

$$
\lambda^{* *}=\frac{\mu K_{1} K_{2} K_{3} K_{4}\left(R_{C}-1\right)}{A_{2}}
$$

So system (1) has a unique (stable) endemic equilibrium if $R_{C}>1$, since $\lambda^{* *}>1$ if $R_{C}>1$.

Local Stability of Endemic Equilibrium Point (EEP) of the Model: The Jacobian expressed in terms of force of infection gives

$$
J\left(\xi^{* *}\right)=\left(\begin{array}{cccccc}
-\left(\lambda^{* *}+\mu\right) & 0 & 0 & 0 & 0 & 0 \\
\lambda^{* *} & -K_{1} & 0 & 0 & 0 & 0 \\
0 & \sigma & -K_{2} & 0 & 0 & 0 \\
0 & 0 & \gamma_{1} & -K_{3} & 0 & 0 \\
0 & 0 & 0 & \gamma_{2} & -K_{4} & 0 \\
0 & 0 & \tau_{1} & \tau_{2} & \tau_{3} & -\mu
\end{array}\right)
$$

Using elementary row-transformation, we have
The Eigenvalues are giving as

$$
\lambda_{1}=-\lambda^{* *}-\mu
$$

from (25) if $R_{C}>1$ then $\lambda_{1}<0$

$$
\begin{aligned}
& \lambda_{2}=-K_{1}<0 \\
& \lambda_{3}=-K_{2}<0 \\
& \lambda_{4}=-K_{3}<0 \\
& \lambda_{5}=-K_{4}<0 \\
& \lambda_{6}=-\mu<0
\end{aligned}
$$

We claimed the following results

Lemma 4: The positive endemic equilibrium state of the model (1) is locally asymptotically stable (LAS) when the control reproduction number, $R_{C}>1$ but close to 1 .

\section{RESULTS AND DISCUSSION}

In this session we carried out the simulation of model (2), examining the effect of treating infectious individuals on different classes using Maple software. Table 1 contains all the values used in simulations.

\begin{tabular}{llll}
\multicolumn{1}{l}{ Table 1: Variable and Parameter Values } \\
\hline \multicolumn{1}{l}{ Variable } & Description & Baseline Values & References \\
\hline$S(0)$ & Susceptible Individuals & $24000 \mathrm{Ind} /$ Year & Assumed \\
$E(0)$ & Latent Individuals & $200 \quad$ Ind/Year & (Andrawus et al.,2017) \\
$\mathrm{I}(0)$ & Infectious Individuals & $100 \quad$ Ind/Year & Assumed \\
$\mathrm{R}_{1}(0)$ & First Line Treatment & $40 \quad$ Ind/Year & Assumed \\
$\mathrm{R}_{2}(0)$ & Second Line Treatment & $50 \quad$ Ind/Year & Assumed \\
$\mathrm{R}(0)$ & Recovered Individuals & $98 \quad$ Ind/Year & (Egonmwan and Okuonghae, 2009) \\
\hline Parameters & Description & Values & References \\
\hline$\Lambda$ & Constant recruitment rate & $200 \quad$ Ind/Year & [8] \\
$\beta$ & Effective contact rate & $10 \quad$ Ind/Year & (Adewale et al.,2009) \\
$\mu$ & Natural mortality rate & $0.02041 /$ year & (Egonmwan and Okuonghae, 2009) \\
$\sigma$ & Progression rate & $0.048 /$ year & (WHO, 2013; WHO, 2016) \\
$\gamma_{1}$ & Default treatment of Infectious Individuals & $(0,1)$ & (Yang et al.,2014) \\
$\gamma_{2}$ & Default treatment of First Line treatment & $(0,1) \quad$ & (Yang et al.,2014) \\
$\tau_{1}, \tau_{2}, \tau_{3}$ & Treatment rates & $(0,1),(0,1),(0,1)$ & -- \\
$\delta_{1}, \delta_{2}, \delta_{3}$ & Disease Induced death rates & $(0,1),(0,1),(0,1)$ & -- \\
\hline
\end{tabular}




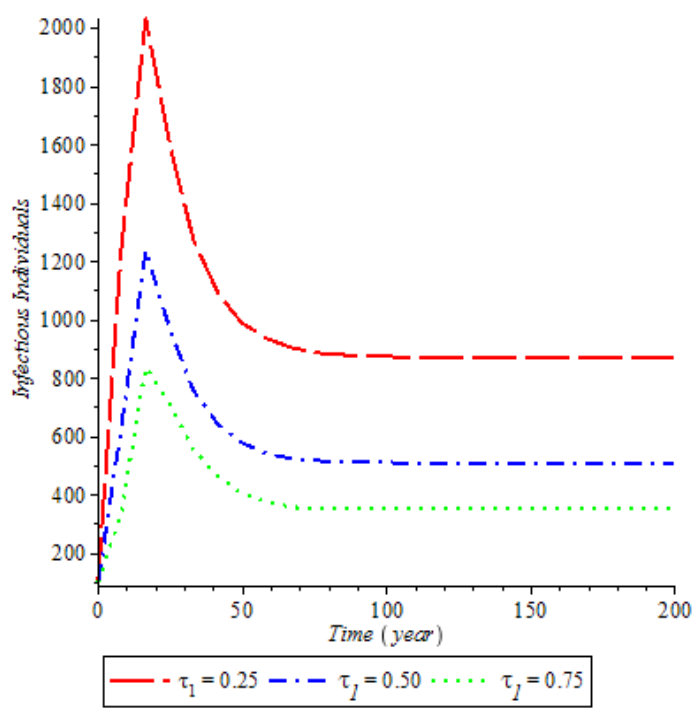

Fig 2: The Effect of Varying Treatment Rates on Infectious Individuals

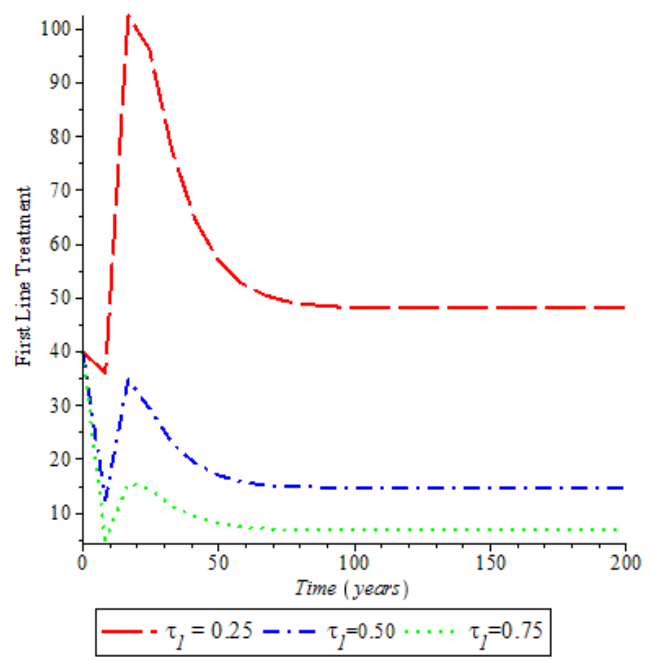

Fig 3: The Effect of Varying Treatment Rates on First Line Treatment

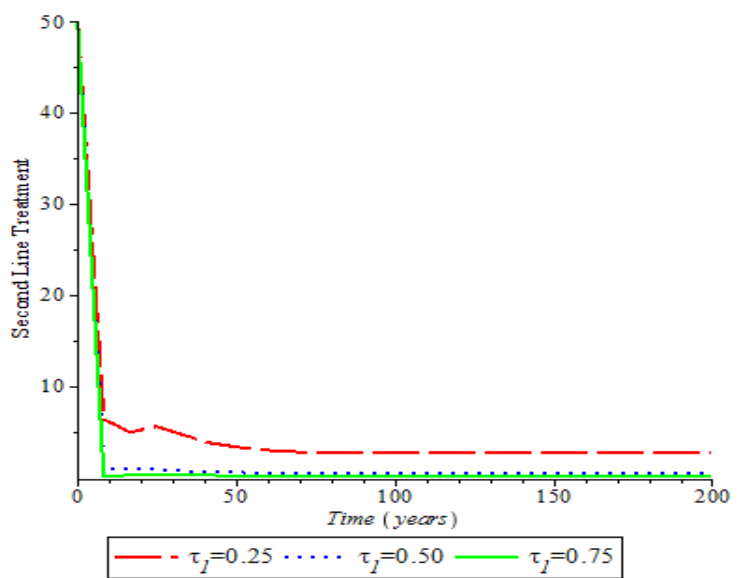

Fig 4: The Effect of Varying Treatment Rates on Second Line Treatment

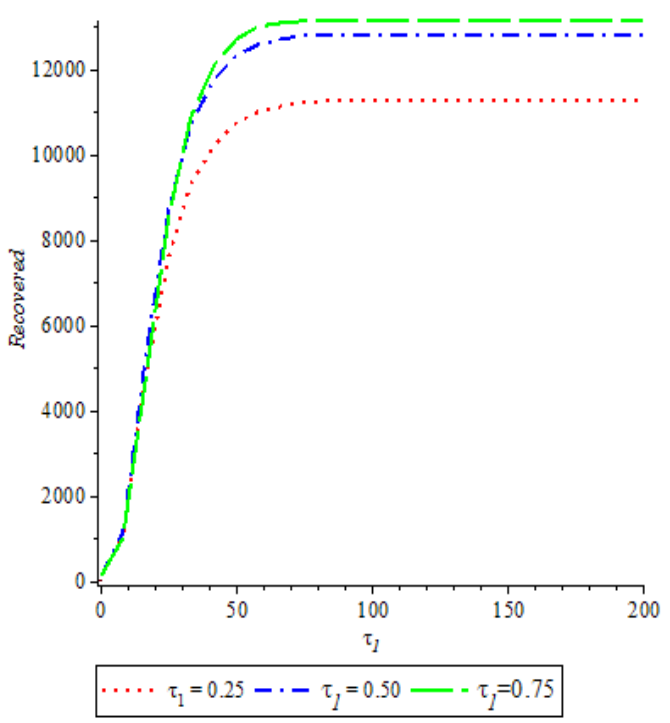

Fig 5: The Effect of Varying Treatment Rates on Recovered Individuals

Figure 2 is showing the effect of varying the treatment rate on Infectious individual, increasing treatment rate leads to reduction of Infectious individuals in a society. Epidemiologically, this will reduced the disease burden in a society. Figure 3 is showing the effect of varying a treatment rates on First line treatment (those that defaulted in treatment which will lead to antidrug resistant), increasing the treatments rates which will leads to fewer case of First line treatment.

Figure 4 shows the effect of varying the treatment rates on Second line treatment (those that defaulted in treatment for the second time which will lead to antidrug resistant), increasing the treatment rates leads to a small number of such cases in a society that is the cases of second line treatment. Figure 5 shows the effect of varying treatment rates on recovered individuals, increasing the treatment rates which will leads to the recovered individual as years goes by.

Conclusion: In this paper, a mathematical model of a tuberculosis transmission dynamics incorporating first and second line treatment was formulated and analyzed in order to gain better understanding of the disease dynamics. The DFE was shown to be locally asymptotically stable when the $R_{C}$ is less than one and the EEP was also shown to be locally asymptotically stable when the $R_{C}$ is greater than one. Numerical simulation shows that treating Infectious Individuals can leads to drastic reduction in second line treatment cases in a society which will also leads to reduction of Tuberculosis (TB) burden in a society. 


\section{REFERENCES}

Adewale, SO.; Podder, CN; Gumel, AB. (2009): Mathematical analysis of a TB transmission model with DOTS. Can. Appl. Math. Quat. 17(1), $1-36$.

Andrawus, J.; Eguda, FY (2017): Analysis of a Mathematical Model to Investigate the Dynamics of Dengue Fever. J. Appl. Sci. Environ. Manage. 21(4), 626-638.

Andrawus, J.; Nwankwo,A. and Okuonghae, D (2017) Bifurcation Analysis of a Mathematical Model For TB-Dengue Co-Infection. Nig. Res. J. Eng. Environ. Sci. 2(2), 390-407.

Aparicio, JP; Castillo-Chavez, C. (2009): Mathematical modelling of tuberculosis epidemics. Math. Biosci. Eng. 6 (2), 209-37

Castillo-Chavez, C.; Song, B. (2004): Dynamical models of tuberculosis and their applications. Math. Biosci. Eng. 1(2), 361-404

Delogu, G.; Sali, M.; Fadda, G. (2013): The biology of mycobacterium tuberculosis infection. Mediterr. J. Hematol. Infect. Dis. https://doi.org/10.4084/MJHID.2013.070

Egonmwan, AO; Okuonghae, D. (2018): Analysis of a mathematical model for tuberculosis with diagnosis. J. Appl. Math. Comput. Springer. https://doi.org/10.1007/s12190-018-1172-1, .

Feng, Z.; Castillo-Chavez, C.; Capurro, AF. (2000): A model for tuberculosis with exogenous reinfection. Theory. Pop. Biol. 57(3), 235-47
Issarowa, CM; Muldera, N; Wood, R. (2015). Modelling the risk of airborne infectious disease using exhaled air. J. Theory. Biol. 372, 100-106

Okuonghae D.; Ikhimwin BO (2016) Dynamics of a Mathematical Model for Tuberculosis with Variability in Susceptibility and Disease Progressions Due to Difference in Awareness Level. Front. Microbiol. 6:1530.

Okuonghae, D. (2013): A mathematical model of tuberculosis transmission with heterogeneity in disease susceptibility and progression under a treatment regime for infectious cases. Appl. Math. Model. 37(10-11), 6786-6808

World Health Organization (WHO) (2014): Guidelines on the management of latent tuberculosis infection

World Health Organization (WHO) (2016): Global tuberculosis report. WHO report.

Yang, W.T., Gounder, C.R., Akande, T., et al. (2014): Barriers and delays in tuberculosis diagnosis and treatment services: does gender matter? Tubercul. Res. Treat. https://doi.org/10.1155/2014/461935

World Health Organization (WHO) (2013): Global tuberculosis report. WHO report 